\title{
Heydorn, Heinz-Joachim
}

\section{Realer Humanismus und humanistisches Gymnasium (1965)}

\author{
Pädagogische Korrespondenz (2005) 34, S. 8-24
}

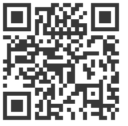

Quellenangabe/ Reference:

Heydorn, Heinz-Joachim: Realer Humanismus und humanistisches Gymnasium (1965) - In:

Pädagogische Korrespondenz (2005) 34, S. 8-24 - URN: urn:nbn:de:0111-opus-79763 - DOI:

10.25656/01:7976

https://nbn-resolving.org/urn:nbn:de:0111-opus-79763

https://doi.org/10.25656/01:7976

in Kooperation mit / in cooperation with:

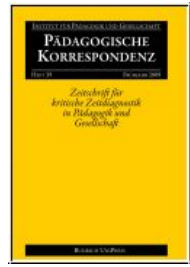

https://pk.budrich-journals.de

\section{Nutzungsbedingungen}

Gewährt wird ein nicht exklusives, nicht übertragbares, persönliches und beschränktes Recht auf Nutzung dieses Dokuments. Dieses Dokument ist ausschließlich für den persönlichen, nicht-kommerziellen Gebrauch bestimmt. Die Nutzung stellt keine Übertragung des Eigentumsrechts an diesem Dokument dar und gilt vorbehaltlich der folgenden Einschränkungen: Auf sämtlichen Kopien dieses Dokuments müssen alle Urheberrechtshinweise und sonstigen Hinweise auf gesetzlichen Schutz beibehalten werden. Sie dürfen dieses Dokument nicht in irgendeiner Weise abändern, noch dürfen Sie dieses Dokument für öffentliche oder kommerzielle Zwecke vervielfältigen, öffentlich ausstellen, aufführen, vertreiben oder anderweitig nutzen

Mit der Verwendung dieses Dokuments erkennen Sie die Nutzungsbedingungen an.

\section{Terms of use}

We grant a non-exclusive, non-transferable, individual and limited right to using this document.

This document is solely intended for your personal, non-commercial use. Use of this document does not include any transfer of property rights and it is conditional to the following limitations: All of the copies of this documents must retain all copyright information and other information regarding legal protection. You are not allowed to alter this document in any way, to copy it for public or commercial purposes, to exhibit the document in public, to perform, distribute or otherwise use the document in public.

By using this particular document, you accept the above-stated conditions of use.

\section{Kontakt / Contact:}

peDOCS

DIPF | Leibniz-Institut für Bildungsforschung und Bildungsinformation Informationszentrum (IZ) Bildung

E-Mail: pedocs@dipf.de

Internet: www.pedocs.de

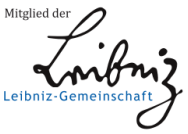




\section{DAS HISTORISCHE LEHRSTÜCK}

\section{Andreas Gruschka}

Heinz-Joachim Heydorn / Herwig Blankertz:

Eine Kontaktaufnahme

8 Heinz-Joachim Heydorn

Realer Humanismus und humanistisches Gymnasium

25 Briefwechsel zwischen Heinz-Joachim Heydorn und Herwig Blankertz

31 Herwig Blankertz

Der Konservative als Revolutionär

\section{SINNBILDER}

Wolfgang Münzinger / Andreas Gruschka

Zusammengefügt und analysiert

Jacques-Louis David malt Antoine und Marie Lavoisier

\section{AUS WISSENSCHAFT UND FORSCHUNG}

Jens Rosch

Mathematik zwischen Dressur und Verstehen

Phänomenologie einer unbehaglichen fachkulturellen Antiquiertheit am Beispiel geometrischer Berechnungen bei PISA

\section{ERZIEHUNG NEU}

Johannes Appel

Vor Gebrauch wird gewarnt!

Wie eine Benutzerordnung für einen Computerraum erziehen soll.

\section{DER REFORMRÜCKSCHLAG}

\section{Günter Rüdell}

Wie Selektion eingeklagt wird - eine Fallstudie 


\section{Realer Humanismus und humanistisches Gymnasium} (1965)

Die Stellung des humanistischen Gymnasiums ist heute im öffentlichen Bewußtsein in einem ganz besonderen Maße wertbezogenen Urteilen ausgesetzt; weithin gilt es als eine Bildungsstätte, in der gegenüber dem Nivellement der funktionalisierten Gesellschaft ein elitäres Bewußtsein erhalten und Bildung aus den unmittelbaren Aufgaben der Gegenwart herausgesetzt wird, um sich selbst Gegenstand zu sein. Soziologisch, so wird argumentiert, sei keine Schicht mehr vorhanden, die eine solche Bildung im Sinne der abgestorbenen leisure class des alten deutschen Bildungsbürgertums noch fruchtbar machen könne, politisch sei sie kaum wünschenswert. Gerade die klassische deutsche Bildung, die das humanistische Gymnasium vertrete, sei der gesellschaftlichen Aufgaben in unserem Lande nicht Herr geworden, sie trage ihren Teil an der „deutschen Daseinsverfehlung“, um mit Ernst Niekisch zu reden, sie habe im besten Falle zu einer Persönlichkeitsbildung beigetragen, die dem Wechsel der Zeitläufe ohnmächtig überlassen blieb. Tatsächlich wird diese Kritik an der humanistischen Bildung kaum jemals so offen ausgesprochen, obgleich sie genuiner Ausdruck vorherrschender gesellschaftlicher Denkweisen ist, weil ihr eine von derselben Gesellschaft entwickelte Sekundärtendenz widerspricht. Diese Seķundärtendenz resultiert aus der Bodenlosigkeit, aus dem Mangel an wirksamer geistiger Kontinuität. In ihrer Nullpunktexistenz bedarf die Gesellschaft einer Rechtfertigung, durch die ihre Ängste neutralisiert werden, sie bedarf der Rückverbindung zu lebensdeutenden Symbolen, um ihren eigenen Abgrund zuzudecken. Somit ist eine gewisse Paradoxie der Situation feststellbar: Während die Faktizität und die mit ihr einhergehenden Verhaltensweisen den Bereich der humanistischen Bildung immer stärker einengen, behält sie doch zugleich noch jenen Wert des schönen Scheins, dessen die Gesellschaft bedarf, um sich auszuhalten. Darin besteht zur Zeit ihre ideologische Restrechtfertigung; auch dies mag bald ein Ende haben. Die allgemeine schulpolitische Tendenz, in der die gesellschaftlichen Veränderungen und somit auch die Veränderungen des gesellschaftlichen Bewußtseins reflektiert werden, weisen kaum auf eine dauerhafte Bewahrung der altsprachlichen Bildung hin. Wesentlich ist dabei gewiß auch die Tatsache, daß die humanistische Bildung im Sinne der europäischen Überlieferung in den großen Industriestaaten, die aus der Entwicklung des 19. und des 20. Jahrhunderts hervorgegangen sind, zu keiner Entfaltung gelangt ist; andere geschichtliche und materielle Voraussetzungen haben eigene Konsequenzen bewirkt. Somit scheint die Entwicklung präjudiziert, die objektive Tendenz ist nicht zu verkennen; schließlich bleibt hinzuzufügen, daß die Ausrottung der alten bürgerlichen Bildungsschichten in unserem Lande, ihre physische, materielle und geistige Liquidierung auch nach einer blutigen Revolution nicht gründlicher hätte sein können. Wie alle Entwicklung, so ist 
auch diese nur wahrscheinlich, nicht zwangsläufig; sie kann modifiziert werden, wenn sich die humanistische Bildung selbst nicht nur unter traditionalistischen Aspekten begreift, sondern ihre Bestimmung neu zu durchdenken sucht. Sie hat sie zu durchdenken im Hinblick auf die konkreten Aufgaben, die die Gesellschaft stellt. Was unter „Aufgabe“ verstanden werden muß, macht die Schwierigkeit des Problems aus. Eines darf jedoch vorweggenommen werden: Jede Konzession an eine landläufige Modernität, an den verdünnten und das kritische Bewußtsein unendlich langweilenden Reflex des Bildungspragmatismus käme einer im Vorwege erklärten Selbstaufgabe der altsprachlichen Bildung gleich. Es steht die Frage nach einer Aktualisierung der geistigen Substanz der humanistischen Bildung; ist sie nicht möglich, bleibt nur der Bezug auf das Gewesene, so sollten die altsprachlichen Gymnasien gleich geschlossen werden.

Realer Humanismus: Dies heißt, welche Bildung die geeignete sei, dem Menschen eine Chance zu geben, in der von ihm geschaffenen Welt Mensch zu sein; in der wahren, der schmutzigen Welt soll der Mensch aus der Verhaftung an die Dinge und die über ihn gesetzte Verfügung zu sich selbst und zum Bewußtsein seiner Freiheit gelangen. Hier ist keine Welt der künstlichen Paradiese gemeint, der Fluchtburgen und der Verstecke vor der Bedrohung, die die Wirklichkeit in sich enthält, sondern die Welt der industriellen Abhängigkeiten und der anonymen Herrschaft. Der unvergleichliche Zauber der antiken Erde, die ästhetische Verwandlung der geistigen Landschaft, führen eher von dieser entscheidenden Frage fort und öffnen jene intermundien, da Epikurs Götter in das Nichts gesetzt sind; es geht um die civitas terrena, um das Spinnengewebe, in dem wir sitzen. Nun soll zu ihr jedoch auf dem Umweg des Geistes geführt werden, über einen Hinweis auf die Wurzeln der neueren Bildungsideologie, im Zusammenhang mit einigen bedeutsamen Stadien des Weges, der zu unserer Gegenwart führt.

Der Gedanke einer realen Bildung im Gegensatz zu den theologisch-ontologischen, später auch literarisch-ästhetischen Wurzeln des Humanismus ist bereits mit der Wende zur Neuzeit gegeben. Der Mensch soll auf eine unmittelbare Weise seinem Menschentum angenähert werden, durch die Erfahrung der wirklichen Dinge. Philosophisch stützt sich diese Bildungstheorie weithin auf den Sensualismus, der Gedanke der Entfaltung des Selbstbewußtseins durch ökonomisch relevante Arbeit tritt früh hinzu. Bei Comenius werden diese Tendenzen in einen überaus eindrucksvollen Zusammenhang gebracht; nicht ohne Abhängigkeit von ihm gewinnen sie im Pietismus historische Geltung. Immer geht es darum, in einem für den damaligen Menschen noch unmittelbar erfahrbaren Lebenskreis das natürliche Licht zur Entfaltung zu bringen, am Gegenständlichen und über das Gegenständliche; früh schon wird die Situation der Massen unter starken sozialpädagogischen Aspekten einbezogen. Die ersten großen bildungspolitischen Maßnahmen in Deutschland, so die Erlasse des bedeutenden Herzogs Ernst von Gotha mitten während des Dreißigjährigen Krieges, lassen diese Gesichtspunkte erkennen; sie wirken weiter in ungewöhnlicher anthropologischer Vertiefung bei Pestalozzi und, über ihre englische Vermittlung durch John Bellers und Owen -, bei Marx. Die Aufklärung bringt diesen Gedanken eines auf die Wirklichkeit hin angesetzten realen Humanismus zu gewichtiger Entfaltung - nicht nur im Bereich der mächtig einsetzenden Elementarbildung, sondern durchaus auch in Ansätzen eines mittleren und höheren Schulwesens, so in der mathematisch- 
ökonomischen Realschule Haeckers und früher noch in den Lehrplänen der Ritterakademien. Es ist in diesem Zusammenhang erwähnenswert, daß aus einer dieser Ritterakademien die Universität Halle, Zentrum des Pietismus und der Aufklärung - in der Person des Thomasius überzeugend verbunden -, hervorgegangen ist. Für diese erste frühe Epoche eines realen Humanismus bleiben die Franckischen Anstalten stellvertretend; der auf die Rettung des Menschen gerichtete Impuls ist unverkennbar. Die Degeneration der überlieferten Bildung, in protestantischen Ländern der durch Melanchthon inaugurierten Gelehrtenschulen, ist zur gleichen Zeit offenbar. In diesen Jahrzehnten scheint es, als ob die tradierte Bildung den starken, humanen Impulsen der Aufklärung nicht standhalten kann und zum Spiegelbild absinkender Lebensordnungen und Inhalte wird. Somit darf festgestellt werden, daß sich die ersten bedeutenden Anstöße eines realen Humanismus gegen die überlieferte Bildung und ihren schwindenden Gehalt richten. Die Wirklichkeit - Pestalozzis „Kot der Welt" -, innerhalb derer sich der Mensch gewinnen soll, ist noch eine den Menschen eng umschließende, sich an ihn schmiegende, auch in ihren Widersprüchen noch reproduzierbare Wirklichkeit; ihre Produktionsweisen sind einsichtig. Die Inbezugsetzung auf das Problem der Humanität kann noch direkt erfolgen. Die Anstöße dieses realen Humanismus vollziehen sich in Deutschland zugleich noch weithin in einer vornationalen Bewußtseinslage und erreichen in ihr ihre Grenzen.

Die Erneuerung der humanistischen Bildung, die der Aufklärung folgt, hat nicht nur im Hegelschen Sinne entscheidende Momente der Aufklärung in sich aufgehoben - in einem weit größeren Umfang, als es ihr in ihrer historischen Position jemals zum Bewußtsein gelangen konnte -, sondern sie ist Teil einer nationaldemokratischen und humanen Bewegung, die zeitweilig den Charakter einer levée en masse annimmt. Die humanistische Bildungsideologie ist nun unlösbar verbunden mit dem Prozeß der nationalen Selbstbewußtwerdung in Deutschland; am Griechentum wird das eigene Selbstbewußtsein im Sinne einer beispiellosen Wahlverwandtschaft erfahren, mit allen Anzeichen des Hoffens auf menschliche Verwirklichung. Es ist dies ein unwiederbringlicher Augenblick tiefer Erregung und schmerzlicher Sehnsüchte, nicht loslösbar von der französischen Revolution und ihren Folgen, von der Römertugend des Konvents, aber eben eingebettet in eine deutsch-griechische Landschaft und durch sie verwandelt. Griechentum ist hier ganz noch Gestalt, Winckelmannisches Maß, fruchtbare Umgrenzung im Selbstverständnis des aufsteigenden Bürgertums; die dunklen und auflösenden Besessenheiten, wie sie später von Nietzsche und Erwin Rohde an der Schwelle des gesellschaftlichen Verfalls aufgedeckt werden, liegen noch außerhalb des Bewußtseins. Dieses klassische Bild des Griechentums ist somit den Ansätzen einer umfassenden gesellschaftlichen und sozialen Revolution im Deutschland des beginnenden 19. Jahrhunderts eng verbunden; die erneuerte Bildung ist Teil dieser Umwälzung. Von dem Grundgedanken dieser Bildung her wird bei Humboldt das Bildungsprinzip der ganzen Nation entwickelt, nicht als fiktive Welt des Geistes, sondern als reale Emanzipation aller, einschließlich der Juden, als Recht auf Menschenwürde, auf die Würde des denkenden Wesens, losgelöst von Notdurft und Selbstentfremdung, als einheitliches Prinzip des gesamten Bildungssystems. Die Bewegung, deren wesentlicher Teil eine erneuerte Ideologie der humanistischen Bildung ist, scheitert als politische Bewegung; die Konsequenzen ihrer Durchsetzung sind im Bereich der 
Bildung nur erahnbar, weil nie realisiert. Eine Argumentation, die das Scheitern der politischen Bewegung vor allem ihrer Bildungsideologie anlastet, beweist nur, daß ihr die entscheidenden historischen Wirkkräfte verborgen bleiben; Deutschland ist nicht am humanistischen Gymnasium gescheitert, selbst wenn es miserabel gewesen wäre. Auf dem Höhepunkt jedoch der am Bilde des Griechentums erfahrenen Selbstbewußtwerdung des deutschen Geistes sind Deutschtum und Menschentum, Nationalität und Humanität auf das innigste miteinander vermählt, der arme und an seine physische Notdurft gekettete Mensch soll Subjekt werden, Teilhaber jener Schönheit, die das Sinnliche mit dem Unvergänglichen verbindet. Erst nach dem Untergang der politischen Emanzipationsbewegung des deutschen Bürgertums bleibt nur die verstümmelte Form des Humanismus zurück, vielfach abgedrängt in eine imaginäre Welt, als Bildungsbürgertum, das dort, wo es nicht kapituliert, vor den heraufkommenden neuen Gewalten eine Möglichkeit ästhetisch-individueller Erlösung sucht. Wenn somit im Hinblick auf unsere Bildungsformen der Versuch unternommen wird, ihren progressivhumanen Charakter zu bestimmen, bleibt der Ausgangspunkt des humanistischen Gymnasiums mit dem bedeutendsten Versuch einer deutschen Selbstfindung verbunden, dessen Gelingen uns die Barbarei erspart hätte, einer Selbstfindung, die den Menschheitsgedanken in sich aufhob unter Einschluß und Transformation der Werte, die die Aufklärung hinterlassen hatte.

Die Festlegung des humanistischen Gymnasiums als einer reaktionären und den zeitgenössischen Aufgaben gegenüber unaufgeschlossenen Bildungsanstalt geschieht daher auch erst spät und unter außerordentlich aufschlußreichen Voraussetzungen. In der Eröffnungsansprache Wilhelm II. anläßlich der Schulkonferenz von 1890 heißt es: "Wer selber auf dem Gymnasium gewesen ist und hinter die Kulissen gesehen hat, der weiß, wo es da fehlt. Und da fehlt es vor allem an der nationalen Basis. Wir müssen als Grundlage für das Gymnasium das Deutsche nehmen; wir sollen nationale junge Deutsche erziehen und nicht junge Griechen und Römer. Wir müssen von der Basis abgehen, die jahrhundertelang bestanden hat ... Wir müssen das Deutsche zur Basis machen «. ' Wie heute, so wird der Schule, vornehmlich jedoch der humanistischen Bildung, vorgeworfen, daß sie die »Bedürfnisse des Lebens« ignorierte; was damit gemeint ist, wird durch den Kaiser selbst interpretiert: "Das Reich ist geeint; wir haben, was wir erreichen wollten, und dabei ist die Sache stehengeblieben. Jetzt mußte die Schule, von der neu gewonnenen Basis ausgehend, die Jugend anfeuern und ihr klarmachen, daß das neue Staatswesen dazu da wäre, um erhalten zu werden.«2 Es geht um die Heranbildung der Jugend »zu den Anforderungen unseres modernen Staatslebens«. Auf diesem, und keinem anderen Hintergrund nun, vollzieht sich die Auseinandersetzung um den Vorrang einer realistischen Bildung, der durch die gesetzliche Begründung von Realgymnasien und Oberrealschulen seinen Ausdruck findet und auch in den Lehrplänen der Volks- und vor allem der Mittelschulen wirksam wird. Die Frage, die im Mittelpunkt steht, ist die Frage nach einer Bildung, die der Epoche der imperialistischen Expansion das wissenschaftliche und ideologische

1 Deutsche Schulkonferenzen - Verhandlungen über Fragen des höheren Unterrichts. Berlin, 4. bis 17. Dezember 1890, Berlin 1891, Reprint Glashütten/Ts. 1972, Band 1, S. 72.

2 Ebd., S. 71. 
Instrumentarium liefern kann. Anläßlich der Schulkonferenz des Jahres 1900 erklärte der preußische Unterrichtsminister Dr. Studt: „Auf der anderen Seite aber läßt sich bei einem Rückblick auf die Konferenz von 1890 nicht verkennen, daß die Allerhöchsten Intentionen nicht überall zur vollen Ausführung gelangt sind. Dies gilt besonders von der angestrebten Erweiterung des realistischen Wissens. Es handelt sich hierbei um eine hochbedeutsame Frage für die wirtschaftliche Weiterentwicklung unseres Volkes, die an Wichtigkeit noch zugenommen hat durch das Wachsen unserer internationalen Beziehungen und durch das weitere Aufblühen der deutschen Seemacht".. Die ideologische Basis der Neubegründung der realistischen Bildung in Deutschland in den Jahren zwischen 1870 und 1914 wird jedoch am deutlichsten an dem Beitrag, den der Erzieher des Kaisers, Prof. Dr. Hinzpeter, zu den Verhandlungen der Konferenz leistet: „Die persönliche geistige Entwicklung galt früher als hohes, erstrebenswertes Ziel, und jetzt gilt sie nur noch als Mittel zu erfolgreicher Betätigung im wilden Kampfe ums Dasein. Man will nicht bloß höhere Lebensanschauung wie damals, man will höhere Lebenshaltung, und zwar die ganze Nation so gut wie der einzelne. Damit hat sich, scheint mir, auch das ganze Bildungsideal bedeutend verschoben. Es gehörte früher zum gebildeten Manne die Kenntnis der alten Sprachen, der antiken Kultur und Geschichte; es gehört jetzt zum gebildeten Manne die Kenntnis der neueren Sprachen, der deutschen Kultur und Geschichte und der Naturwissenschaften"."

Obwohl es der humanistischen Bildung auch nach 1870 gelingt, sich im Hinblick auf eine jahrhundertealte Tradition wesentliche Reservate zu erhalten, steht doch die Entwicklung ganz im Schatten andauernder Rückzugsgefechte; sie wird auf Grund des ihr wesenseigenen Stoffes als untauglich angesehen, den wahren Bedürfnissen der Zeit zu dienen. Die geistesgeschichtliche Situation wird rückblickend überaus deutlich: Während das durch den Neuhumanismus geprägte klassische Gymnasium auch die Hinterlassenschaft der Aufklärung in sich einbegriff, hatte die Neufundierung der realistischen Bildung jeden Zusammenhang mit ihren ersten humanen Impulsen in unserer Geschichte verloren. Ihre Ideologie erweist sich als primitiv materialistisch, sozialdarwinistisch, präfaschistisch. Text und Kontext der Zeit machen dies offenbar.

Die Wertung von Bildungseinrichtungen hat in ihrem konkreten Bezugssystem zu erfolgen; sie wollen in ihrer geschichtlichen Relevanz begriffen sein. Eine Analyse der Vorposition unserer Gegenwart, und daher auch für sie von unmittelbarer Bedeutung, macht einsichtig, daß die unreflektierte Identifikation der humanistischen Bildung mit reaktionären Geschichtsmomenten in keiner Weise haltbar ist, sie ist im besten Falle Ausdruck eines mechanistischen Fortschrittsglaubens, der die Erfahrungen dieses Jahrhunderts nicht zur Kenntnis genommen hat und daher ernsthaft nicht diskutierbar ist. Der reale Pragmatismus des Willens zur Macht, der die Entfaitung von Industrie und Technik unter seine Interessen zwingt und in einen perversen Bildungsfunktionalismus übersetzt, begann der alten Bildung überdrüssig zu werden; die Gründe werden zu untersuchen sein. Es ist daher nur natürlich, daß auch unter der späteren Herr-

3 Deutsche Schulkonferenzen - Verhandlungen über Fragen des höheren Unterrichts, Berlin 6. bis 8. Juni 1900, Haile 1902, Reprint Glashütten/Ts. 1972, Band 2, S. 1.

4 Ebd., S. 3. 
schaft des Nationalsozialismus die Skepsis gegenüber der altsprachlichen Bildung erhalten bleibt, war er doch direkte Fortsetzung dessen, was ideologisch bereits vor Ausbruch des ersten Weltkrieges vorbereitet wurde. Mag man dem humanistischen Gymnasium vorwerfen, daß es sich den Problemen des gesellschaftlichen und geistigen Verfalls nicht gestellt hat, daß seine Welt immer fiktiver und isolierter wurde, da $\beta$ viele seiner Vertreter wie Wilamowitz dem Nationalismus nicht minder verfielen als andere - hier muß dann aber auch Werner Jaeger genannt werden, der später das Dritte Reich aus Gründen seiner humanen Überzeugung verließ -, das Mißtrauen der Herrschenden war nicht ohne Grund. Es beruhte auf objektiven Einsichten. Mit der Überwindung der Blut- und Opfermythen, von vorzeitlichen Ängsten hatte die hellenische Kultur als Bewußtseinskultur begonnen, als Zeugung der Gestalt; mit der Auflösung in das Gestaltlose, Dunkle und Vorrationale endete die Welt der bürgerlichen Kultur in Deutschland.

Es ist auf diese Tatbestände hingewiesen worden, um die Ausgangspositionen zu klären, um zunächst einmal Urteile kritisch zu analysieren und auf ihren Wahrheitsgehalt zu befragen. Die Auseinandersetzung um den Wert der humanistischen Bildung seit der Begründung des deutschen Einheitsstaates ist somit nicht, zumindest jedoch nicht primär, durch den Antagonismus zwischen einem liberalen, aufgeklärten und an den Naturwissenschaften orientierten Denken und den brüchig gewordenen Inhalten einer in ihrem Selbstbewußtsein zerstörten aristokratisch-bürgerlichen Schicht charakterisiert. Sie beweist zwar die Verschüttung der bedeutenden frühen Ansätze eines humanen und nationalen Selbstverständnisses, aber an ihre Stelle tritt keine neue Humanität, die sich inhaltlich rechtfertigt. Vielmehr konnte die realistische Anpassung weiter Teile des deutschen Schulwesens an die veränderten, durch die Entwicklung von Industrie und Technik bedingten Umstände zwischen 1870 und 1914, im Zusammenhang mit den allgemeinen gesellschaftlichen Bedingungen, zu eben jenem konfliktlosen Nebeneinander von naturwissenschaftlicher Effektivität und prähistorischer Ideologie führen, das die nachfolgende deutsche Geschichte kennzeichnet. Der Mensch vermag lange in einem Zustand objektiver Bewußtseinsspaltung zu leben und sich dabei wohl zu fühlen, sofern seine Fähigkeit zur Reflexion genügend abgeschwächt ist. Die Forderung einer Anpassung der Bildungsinhalte an die unmittelbare Praxis der Gesellschaft, an die Bedingungen ihrer Übersetzbarkeit in registrierbare Produktion, an konkret bestimmbare Zwecke, spiegelt daher, soweit ihr humaner Charakter in Betracht kommt, den jeweilig qualitativen Inhalt der Gesellschaft, hier ihre Paralyse, höchst augenfällig wieder, sie ist ein Reflex; je unmittelbarer die Interessenverknüpfung zwischen Bildungsinhalten und aktuellen gesellschaftlichen Belangen in Erscheinung tritt, desto mehr wird auch der Bildungsinhalt der Ideologisierung unterworfen. $\mathrm{Ob}$ die ursprünglich humanen Impulse einer realistischen Bildung in Deutschland heute stärker zur Geltung gebracht werden können als in der vorhergehenden Epoche, steht dahin. Die Beantwortung dieser Frage müßte eine Analyse der demokratischen Substanz unseres gesellschaftlichen Lebens einschließen.

Nun kann man natürlich argumentieren, daß die Meriten der klassischen Bildung für die Vergangenheit unbestreitbar seien, daß sie aber dennoch den Anforderungen der Gegenwart nicht zu entsprechen vermöge, weil sich die objektiven Bedingungen unserer Existenz in relativ kurzer Frist stärker verändert hätten als in einer vorherge- 
henden, jahrhundertelangen Entwicklung. Es ginge darum, gänzlich neue Maßstäbe zu finden. Die alte, überschaubare Welt sei zerstört, der Mensch sei in Produktionsformen eingeschlossen, die er nicht rationalisieren und damit auch nicht humanisieren könne, das gleiche gelte für seine größer werdende, der industriellen Manipulation ausgelieferte Freizeit. In das Koordinatensystem der Zwänge sei ein jeder rettungslos einbezogen, es gäbe keine geistige Welt, die von ihm abstrahierbar sei, um eine eigene Substanz gegenüber der Wirklichkeit zu bilden. Das Verhältnis der klassischen Welt des Geistes zu dieser Wirklichkeit sei inkommensurabel, die soziologischen Bedingungen der durch sie geformten Autonomie des Menschen seien zerstört. Von der Bildung müsse die gegenwärtige Gesellschaft einen mobilen Intellekt und eine Variationsbreite von Anpassungsmöglichkeiten fordern, die Beherrschung ihrer sprachlichen und technischen Medien, schließlich die Hinführung zu ihrer produktiven Basis. Nur unter Akzeptierung dieser Bedingungen könne überhaupt die materielle Existenz der Gesellschaft gesichert bleiben und damit auch die Überlebensfähigkeit des einzelnen; nur so könne schließlich der Mensch in seinem eigenen Gehäuse ein Licht anzünden. Die volle Hineinsetzung des Menschen in seine Wirklichkeit sei zugleich auch seine einzige Chance; wir könnten nur diesen Versuch unternehmen oder scheitern. Die Grundthese, die mannigfaltigen Modifikationen unterliegt, dürfte im Kern richtig dargestellt sein; sie erhebt den Anspruch auf Humanität und ist Ausdruck einer immer enger werdenden Verflechtung von Ökonomie und Bildungswesen. Demokratie und Produktion sollen in gleicher Weise befördert werden; sie impliziert die Rationalisierbarkeit der Welt über die Öffnung unmittelbarer Bezüge. Schließlich aber gibt sie vor, einer evident sachlichen Notwendigkeit zu entspringen und wird damit als entideologisierter Anspruch charakterisiert; sie versucht zugleich den Eindruck einer Wiederaufnahme realhumanistischer Tendenzen, die in unserem Lande mit dem Ende der Aufklärung weithin abbrachen, zu erwecken. Bevor jedoch die einzelnen Aspekte ihrer Verwirklichungsmöglichkeit im Zusammenhang mit dem gegenwärtigen Bildungsauftrag des humanistischen Gymnasiums analysiert werden, muß die Frage nach ihren ideologischen Wurzeln gestellt sein. Für unsere Zeit ist die mittelbare Steuerung des Bewußtseins charakteristisch; die Motivationen sind oft schwer erkennbar. An die Stelle der direkten Herrschaft ist weithin eine indirekte getreten, nicht minder effektiv, unter Verschleierung ihrer materiellen und ideologischen Ansprüche. Im Lichte eines kritischen Bewußtseins jedoch erweist sich die vorgegebene reine Sachlichkeit, die Evidenz des Realitätszwanges, als keineswegs voraussetzungslos, sondern als Konsequenz von Voraussetzungen. Sie sind Ausdruck gesellschaftlicher Herrschaftsinteressen und ihnen adäquater geistiger Inhalte. Der Bildungspragmatismus, der die gegenwärtige bildungspolitische Situation in unserem Lande nach Jahren der Erstarrung im Gefolge des Zusammenbruches zu beherrschen beginnt, ist sehr wohl ideologisch vermittelt; ihm unterliegt die Vorstellung einer funktionsgerechten, harmonisierten und ihre Widersprüche auflösenden Gesellschaft. Seine wesentlichen Anstöße kommen aus einer in den Vereinigten Staaten seit Ende des Bürgerkrieges, vor allem aber seit der Jahrhundertwende entwickelten Bildungsideologie; sie gewinnen zusätzliche Bedeutung durch die Mimikry einer Nation, die das Trauma ihrer eigenen Erfolglosigkeit nicht überwunden und mit ihrer eigenen Geschichte zu keiner relativen Übereinstimmung gelangt ist. Die Wurzeln dieser Bil- 
dungsideologie finden sich in den englischen Evolutionstheorien des 19. Jahrhunderts; ihre typische amerikanische Ausprägung ist mitbedingt durch die besondere Lage, in der sich die amerikanische Gesellschaft, nicht zuletzt durch die anschwellenden Immigrationswellen, befand. Die Denkart, die im Behaviourismus ihren charakteristischen Ausdruck findet, mißt der Fähigkeit des „social adjustment“ entscheidende Bedeutung zu, einer Fähigkeit der Einfügung in das Funktionssystem der Gesellschaft unter den Bedingungen schnell steigender industrieller Produktivität. Elemente der Verhaltensbeobachtung, wie sie aus der Tierwelt gewonnen werden, spielen dabei eine wesentliche Rolle; wertrelativistische Tendenzen sind im Hinblick auf die Erfolgskriterien unverkennbar. Die vollkommene Einordnung des Einzelnen in den Sozialkörper wird zum Zeichen seiner vollkommenen Glückseligkeit; der Außenseiter wird zur psychopathischen Erscheinung. Die große amerikanische Literatur dieses Jahrhunderts hat die vorherrschenden Elemente der Denkungsart zum Gegenstand unerbittlich kritischer Analyse gemacht und die Gefahr angezeigt, daß der amerikanische Traum als Karikatur des Menschentums endet. Wohl erscheint nun diese Bildungsideologie angesichts andersartiger historischer Voraussetzungen humanisiert und demokratisiert, jedoch darf die bedeutende liberale Kontinuität der amerikanischen Gesellschaft nicht darüber hinwegtäuschen, daß ihre geistesgeschichtlichen Elemente die gleichen sind, die, über die Vermittlung des Darwinismus, unter den Bedingungen der deutschen Gesellschaft die Vorfeldsideologie des Faschismus bereiteten. Nur unter den Voraussetzungen andauernden gesellschaftlichen Erfolges vermag jene naive Zuordnung von kollektiver Anpassung, Industrialisierung, Humanität und Progreß die in ihr enthaltene Gefahr des Umschlages in die Barbarei zu bannen; ihre grundsätzliche Problematik bleibt in jedem Falle bestehen.

Das Bewußtsein, die unendliche qualitative Differenz des Menschen von der Natur, unterliegt der Auflösung in das produzierende Ganze, es gewinnt keine eigene Substanz. „Way of life“ ist Gegensatz zur Erkenntnis, die ihre Freiheit zum Handeln aus der Distanz gewinnt. Vor diesem Hintergrunde nun müssen die Voraussetzungen, auf denen die Bildungstheorie des humanistischen Gymnasiums zu beruhen hat, präzisiert werden. Es muß dabei im Vorwege gesagt sein, daß diese Voraussetzungen naturgemäß nicht auf die altsprachliche Bildung begrenzt sein dürfen; sie sind jedoch in den Kern dieser Bildung eingeschlossen, sie sind Teil ihres Begriffs. Im Vorwege muß gleichfalls ein Argument aufgenommen werden von dem bereits die Rede war, weil es von beträchtlicher ethischer Relevanz ist: Das Argument, die altsprachliche Bildung könne den Anforderungen der industriellen Produktivität nicht gerecht werden und stünde somit im Widerspruch zu einer wohlverstandenen Selbsterhaltung. Die physische Selbsterhaltung des Ganzen ist Voraussetzung aller Vermenschlichung. Es läßt sich jedoch rein empirisch feststellen und wird durch die Erfahrungen bedeutender Gelehrter unterstützt, daß die altsprachliche Bildung die Erkenntnisfähigkeit im Bereich der Naturwissenschaften nicht abschwächt; eher dürfte auf Grund ihrer Diszipliniertheit das Gegenteil der Fall sein. Das kurze Eingehen auf diese Argumentation geschieht nicht aus pragmatischen Erwägungen, sondern aus Gründen, die keine verbindliche Betrachtungsweise auslassen kann.

Das Menschenbild des klassischen Humanismus versteht sich über das Bewußtsein, über eine durch das Bewußtsein vermittelte, mögliche Autonomie des Geistes, 
in die ein qualitativ bedeutsames Spannungsverhältnis zwischen Welt und Mensch, Subjekt und Objekt notwendig einbezogen bleibt als Bedingung der Fruchtbarkeit. Das Bewußtsein ist nach Augustin der einzige Anknüpfungspunkt zwischen Gott und dem Menschen. Bewußtsein heißt, in der fortdauernden Bedeutung der Ausgangsthese, alle neuentdeckten, oftmals grenzenlos erscheinenden Räume des menschlichen Innern und der umschließenden Welt in die Vergegenständlichung hineinzunehmen, um Freiheit möglich zu machen. Das Unbewußte und Unerkannte wird zur Aufgabe des Bewußtseins, es will von ihm verantwortet sein. Der Versuch des Menschen, sein Leben über Erkenntnis in sein Eigentum zu nehmen - Erkenntnis auch als Aufhebung, als gestaltende Verwandlung des Reichtums der menschlichen Empfindung -, damit Bildung als Bewußtseinsbildung in aller Strenge, gegenüber jedem nur voluntaristischen, vitalistischen oder auf ein gegebenes Funktionssystem bezogenen Ansatz kennzeichnet das Selbstverständnis der Antike. Erst unter den Bedingungen dieses Selbstverständnisses vermag Freiheit zum Problem zu werden als notwendige Vorstufe ihrer Verwirklichung. Bewußtsein schafft Distanz. Alle klassische Bildung beruht auf dem Distanzbegriff; er ist im anthropologischen Grundverständnis angelegt. Indem das Bewußtsein zum konstituierenden Element wird, ist das Gegebene seiner Fraglosigkeit entkleidet, es erzeugt Widerspruch. Die Scheinharmonie entfällt; Harmonie wird Aufgabe über widerspruchhaftes Reifen zur vollen Verwirklichung. Ihre ästhetische Antizipation ist noch keine Wirklichkeit, sondern vielmehr Erinnerung an sie, Erweckung des Gedächtnisses, in der Form vorweggenommene Erfüllung des Inhalts. Weder von der Verhaltenswelt des Tieres noch von der formalen und widerspruchslosen Rationalität der industriellen Organisation führt ein erschließender Zugang zu diesem Selbstverständnis des Menschen; es bleibt außerhalb.

Ist die Bewußtheit des Menschen Ausdruck seines qualitativen Andersseins, so gewinnt dieser antike Gedanke, der vor allem auch die Mächtigkeit des Begriffes, einer Unterwerfung der zufälligen Erscheinung unter das Abstrakte in sich befaßt, erst über die jüdisch-christliche Überlieferung seine introspektive Dimension. Hier erst werden das Allgemeine und das Besondere miteinander verbunden, Icherfahrung als das zunächst nur Zufällige, Vorübergehende, dem Tod Preisgegebene, und die im Augenblick wohnende Dauer. In der Konsequenz bedeutet dies, daß nun erst der einzelne in seine volle Verantwortung genommen wird, daß das Wahre und Allgemeine auch das Individuelle ist, nicht nur im Sinne der Teilhabe, sondern der ganzen, erfahrenen Existenz. Der Mensch wird beim Namen gerufen. So wird eine Verinnerlichung möglich, die den Bereich der Selbstanalyse erschließt und die Frage nach der Rechtfertigung des Leidens stellen kann, um damit den im Bewußtsein vorhandenen Widerspruch, den Widerspruch zwischen der Logik der Vernunft und der Irrationalität, dem kaum gebändigten Chaos des Seienden, als schöpferisch zu begreifen. Im Gewissensbegriff findet diese Verbindung von Individuellem und Überindividuellem, Vorübergehendem und Bleibendem, des Einzelnen und des Ganzen ihre höchste Ausprägung; Senkung des Lotes in einen Brunnen ohne Grund, aber auch Helligkeit, Prüfung an der Norm, an der unverletzbaren Würde aller. Klassische Bildung beruht auf beiden Ausgangspunkten der Tradition, ihrer unlösbaren Verbindung.

Sicher sind die knappen Akzente, die hier gesetzt worden sind, nicht unanfechtbar; obgleich die angesprochenen Elemente der klassischen Bildung substantiell zuge- 
hören, so sind doch auch andere, dem gleichen Boden verbundene denkbar. Die Akzente aber, die heute gesetzt werden müssen, schließen die Bedürftigkeit des Wirklichen ein, sie erhellen jene Aspekte der Überlieferung, deren der gegenwärtige Mensch bedarf. Alle große geistige Wirklichkeit ist in diesem Sinne immer aufs neue präsent. Schließlich aber bleibt das didaktische Prinzip der klassischen Bildung von hervorragender Bedeutung. Die anthropologische Voraussetzung, Bewußtsein als distanzierendes Moment, wird, umgesetzt, zum didaktischen Kriterium; Stoff und Begriff werden dem Unmittelbaren entzogen. Eine auf Distanz beruhende Didaktik erscheint zunächst als konservativ, für das naive Bewußtsein entzieht sie den Menschen der Aufgabe seiner Gegenwart. Hegel, im deutschen Bewußtsein ein konservativer Denker, Vertreter des preußischen Staatslogos, im internationalen Bewußtsein jedoch der Philosoph des 19. Jahrhunderts, der den folgenschwersten intellektuellen Anstoß der Revolution gegeben hat, formuliert dieses Prinzip der Fremdheit, auf dem das humanistische Gymnasium beruht, in seiner Gymnasialrede vom 29. September 1809 in einer außerordentlich bemerkenswerten Weise: „Um aber zum Gegenstande zu werden, muß die Substanz der Natur und des Geistes uns gegenübergetreten sein, sie muß die Gestalt von etwas Fremdartigem erhalten haben. - Unglücklich der, dem seine unmittelbare Welt der Gefühle entfremdet wird; - denn dies heißt nichts anderes, als daß die individuellen Bande, die das Gemüth und den Gedanken heilig mit dem Leben befreunden, Glaube, Liebe und Vertrauen, ihm zerrissen wird! - Für die Entfremdung, welche Bedingung der theoretischen Bildung ist, fordert diese nicht diesen sittlichen Schmerz, nicht das Leiden des Herzens, sondern den leichteren Schmerz und Anstrengung der Vorstellung, sich mit einem Nicht-Unmittelbaren, einem Fremdartigen, mit etwas der Erinnerung, dem Gedächtnisse und dem Denken Angehörigen zu beschäftigen."s Entfremdung ist somit Voraussetzung aller theoretischen Bildung, Bedingung einer ersten Selbstvergegenwärtigung des Geistes; die reale Entfremdung, das zerrissene Bewußtsein, der Widerspruch des Wirklichen, bleiben noch heraus. Dennoch ist die Inbezugsetzung beider Bereiche trotz ihrer gleichzeitig vorgenommenen Abgrenzung nicht ohne Bedeutung; das die Entfremdung vermittelnde didaktische Prinzip führt an die Wirklichkeit als reale Entfremdung heran, es bereitet die Voraussetzungen, unter denen ihre Logik verstanden wird. Wie immer bei Hegel, hüllt sich das revolutionäre Prinzip ein. Das gleiche didaktische Prinzip aber, auch in der geistigen Fortsetzung der Hegelschen Anstöße, wird bei Brecht, in der modifizierten Anwendung für das Theater, zum einzig möglichen Prinzip einer revolutionären Veränderung des Bewußtseins. Im „Kleinen Organon für das Theater“ wird eine dafür bedeutsame Begriffsbildung vollzogen: „Es ist der unwissenschaftliche Blick, der das Fremde als bizarr stempelt. Die neuen Verfremdungen sollten nur den gesellschaftlich beeinflußbaren Vorgängen den Stempel des Vertrauens wegnehmen, der sie heute vor dem Eingriff bewahrt."6 Distanz wird aufgenommen, um ein Vertrautes fortzunehmen, da es Veränderung erschwert. Wenig später heißt es dann überaus prägnant: „Allenthalben treffen wir auf etwas, das zu selbstverständlich ist, als daß wir uns bemühen müßten, es zu verstehen. Was sie miteinander erleben, scheint den Menschen das gegebene

5 Hegel, Georg Wilhelm Friedrich: Sämtliche Werke in 20 Bänden, Frankfurt 1970, Bd. 4, S. 320.

6 Brecht, Bertolt: Schriften zum Theater, Frankfurt 1957, S. 150. 
menschliche Erleben. Das Kind, lebend in der Welt der Greise, lernt, wie es dort zugeht. Wie die Dinge eben laufen, so werden sie ihm geläufig. Ist einer kühn genug, etwas nebenhinaus zu wünschen, wünschte er es sich nur als Ausnahme. Selbst wenn er, was die „Vorsehung“ über ihn verhängt, als das erkennte, was die Gesellschaft für ihn vorgesehen hat, müßte ihm die Gesellschaft, diese mächtige Sammlung von Wesen seinesgleichen, wie ein Ganzes, das größer ist als die Summe seiner Teile, ganz unbeeinflußbar vorkommen - und dennoch wäre das Unbeeinflußbare ihm vertraut, und wer mißtraut dem, was ihm vertraut ist? Damit all dies viele Gegebene ihm als ebensoviel Zweifelhaftes erscheinen könnte, müßte er jenen fremden Blick entwickeln, mit dem der große Galilei einen ins Pendeln gekommenen Kronleuchter betrachtete." Das Prinzip der Distanz enthält den Zweifel am jeweils Gegebenen und an der gegebenen Herrschaft; über Distanz wird auch der Schwindel jener Unvermitteltheit und Ideologiefreiheit erkannt, die sich auf eine angeblich wertneutrale Empirie stützen, und, über die Anknüpfung an ein Vertrautes und Gegenwärtiges, nun erst Ideologisierung umfassend zur Geltung zu bringen vermögen, indem sie seine analytische Hineinnahme in das kritische Bewußtsein unsäglich erschweren. Das didaktische Prinzip der Distanz ist das genuin demokratische Prinzip, weil es die Erscheinungen ihrer Unmittelbarkeit entkleidet, um an ihnen den Antagonismus der Existenz, zunächst fern von allem Verdunkelnden, Leidenschaft und Triebe unmittelbar Affizierenden, zu vergegenwärtigen; es bereitet damit das Vorfeld sinnbezogenen und freien menschlichen Handelns, es hilft Geschichte als humane Möglichkeit offenzuhalten.

Die klassische Bildung vermag die Möglichkeiten, die das Prinzip der Fremdheit an die Hand gibt, vielfältig zu nutzen. Sie nutzt sie vor allem dadurch, daß sie die Abstraktionsfähigkeit des heranreifenden Menschen entwickelt. Loslösung von der verwirrenden Vielfalt bedeutet, den Menschen, ohne Verlust seiner Individualität, wie dargetan, unter den Begriff zu stellen und damit Zuordnungen möglich zu machen. Abstraktionsvermögen und humane Verbindlichkeit gegenüber dem Ganzen sind eng aufeinander bezogen; an jedem Rechtsbegriff, vornehmlich aber an der Entwicklung des römischen Rechtes als einer ersten umfassenden Sicherung des Menschen durch rationale Rechtsordnung, kann dies verdeutlicht werden. Auf dem Hintergrunde noch unentwickelter empirischer Daten werden die entscheidenden Begriffe menschlichen Selbstverständnisses in beispielloser Form entwickelt; Heraklit und Parmenides, Werden und Sein, Platon, Aristoteles, Demokrit, Idealismus, Entelechie, Materialismus, Altes und Neues Testament, Bund, gegenwärtiger, kommender Äon, Messianismus und Eschatologie. Jede Denkform, jede Zuordnungsform wird in ihren großen Ansätzen, in ihren ersten, archaischen Abstraktionen mitgegeben, eben befreit vom Mythos und dunkler Verhaftung, in ihrer reinen Begrifflichkeit; die Fülle des Gegenwärtigen kann nun unter die Forderungen des Geistes gestellt, die Vielfalt in die einigende Kraft des Bewußtseins genommen werden, helle Entscheidung des Menschen wird möglich als Ausdruck bewußter Wahl. Nun mag an dieser Stelle eine Kritik, nicht unberechtigt, darauf hinweisen, daß die Abstraktionen, gerade weil sie neben den Grundkategorien der Natur vor allem diejenigen der Humanität enthalten, das Verständnis der realen gesellschaftlichen Widersprüche erschweren können, indem sie die Fiktion nähren, 
als ob sich alle essentielle Wirklichkeit des Menschen nur innerhalb seines Bewußtseins vollzöge. Die Erkenntnis des realen Widerspruchs innerhalb der Gesellschaft bleibe weithin ausgeschlossen. Das Prinzip der Distanz aber vermag gerade auch diese Kritik aufzunehmen und damit der Gefahr der Idealisierung entgegenzuwirken, der die klassische Bildung leicht unterliegt. In den Stoff der altsprachlichen Bildung sind seit jeher historische Modelle in erheblichem Umfange einbezogen, schon die Auswahl der Schriftsteller macht dies deutlich. Am in die Distanz gerückten historischen Modell, bei Livius, Tacitus, Sallust, vor allem jedoch bei Thukydides, der diese Möglichkeit am unmittelbarsten und tiefgründigsten aufschließt, sollten die realen Probleme der Gesellschaft weit mehr als bisher in ihrer exemplarischen und bleibenden Bedeutung aufgewiesen werden: Widerspruch von Macht und Ohnmacht des Geistes, der materiellen Interessen und der sie repräsentierenden Klassen, der staatlichen Positionen, von Leidenschaft und Vernunft, Autonomie und Entfremdung des Menschen. An keiner Stelle kann dies so geschehen wie hier und zum Ausgangspunkt der Analyse unserer eigenen Gesellschaft und des über sie hinausführenden Menschheitsproblems werden. Die veränderte historische Situation und die andersartigen soziologischen Daten unserer Gegenwart unterliegen, wenn auch mit anderen Inhalten, den gleichen Spannungsmomenten des Wirklichen, innerhalb derer die Humanität aus ihrer Verdunkelung entbunden werden soll. Auch hier wird ein Kompaß an die Hand gegeben; der distanzlose Stoff vermag dies, weil unmittelbarer Ausdruck bestehender gesellschaftlicher Interessen, kaum zu tun.

Es bleibt als letztes die Sprache, die dieses Prinzip der Distanz augenfällig macht. Sprache ist hier nicht nur Ausdruck von Inhalten, so wichtig dies ist, nicht nur Medium der Aussage. Auch sie ist Modell, beispielhafte Weise der Seinsannäherung. Die lateinische, die griechische und die leider zu wenig gepflegte hebräische Sprache ergänzen sich dabei auf das glücklichste. Die Präzision des Lateinischen, oft genug gepriesen, seine Logizität und definitorische Klarheit enthalten eine Qualität, die nun wiederum durch das Griechische ihre notwendige Ergänzung erfährt. Vieldeutig, im Abstrakten noch sinnlich, im Konkreten das Unausgesagte verbergend, zu Hause im Nest der Träume und in der Helle des hellenischen Tags, von bebender Sensibilität und doch auch Härte des Begriffs, in allem Morgenröte des Geistes, enthält es als Muster jene Welt, die dem Lateinischen fehlt. Das Hebräische schließlich spröde und archaisch, dicht, erratischen Blöcken gleich. Zusammen verdeutlicht dies Sprache überhaupt im Bereich unserer Kultur, volle Entfaltung der Vorstellung und des Gefühls, von Denken und Empfindung, über den Vorgang der Bildung. Es darf an dieser Stelle, abweichend von der Thematik, empfohlen werden, als moderne Pflichtsprache des humanistischen Gymnasiums in aller Regel das Französische anzubieten. Es schließt nicht nur an das Lateinische glücklich an, sondern es vermittelt auch, mehr als das Englische, das seinen insularen Charakter nie ganz abgestreift hat, jene für die kontinentaleuropäische Entwicklung hochbedeutsamen Inhalte, wie sie die Aufklärung und ihre Folgen charakterisieren; das europäische Bürgertum findet sich in ihr auf der Höhe seiner Aufgabe. Englisch und Russisch sollten dagegen in jedem Falle - dem Hebräischen gleich -, wahlfrei angeboten werden.

Realer Humanismus war Frage nach der Möglichkeit des Menschen in seiner Welt, der faktischen Welt, in der er zu leben hat, Mensch zu sein. Es ging zugleich um die 
Frage nach jener Bildung, die Hilfe anbieten kann. Die Realität dieser Welt ist durch die Entfesselung der Produktivkräfte charakterisiert; bei fortschreitender Rationalisierung können die materiellen Möglichkeiten des Menschen eine unerhörte Steigerung erfahren. Die objektiven Bedingungen der industriellen Arbeit und die zwangsläufig größer werdende internationale Verflechtung der Ökonomie, die immer umfassendere Räume zur Produktionsentfaltung benötigt, schaffen - die Gefahr eines kollektiven Selbstmords für einen Augenblick ignoriert -, Verhaltensweisen, die jenseits aller ideologischen Differenzen die einzeinen Gruppen der Menschheit immer stärker aneinander angleichen werden. Angesichts des widersprüchlichen Gefälles der Entwicklung enthält dieser Vorgang Momente tödlicher Gefahr und den Antagonismus primärer und sekundärer Tendenzen, die einander ausschließen. Die rapide Einbeziehung der ganzen Menschheit in einen Prozeß weckt uralte Sehnsüchte der Vereinigung und Angst vor ihrer Realisation als totalem Sinnverlust. Die Gesellschaft, der der einzelne in den Industrieländern verhaftet ist, erscheint rational, da sie auf der Widerspruchslosigkeit naturwissenschaftlicher Erkenntnis basiert; von hier aus wird die innere Verbindung von Industrieentwicklung und optimistischer Lebenserwartung erklärbar, wie sie das 19. Jahrhundert entwickelt hatte, die Fiktion einer glückbringenden Übereinstimmung von Bewußtseinsgesetz und Wirklichkeit. Der alte Rationalismus, mit seiner Forderung nach Übereinstimmung von Sein und Denken, sah eine Möglichkeit der Erfüllung. Die Erfahrung des Wirklichen aber hat Korrektive gesetzt. Wohl ist die Gesellschaft ihrer Form nach rational, aber ihr innerer Zusammenhang sowie die Art ihrer Steuerung sind kaum einsehbar; ein Teilstück des Kanalisationssystems kann erkannt werden, die Ordnung des Ganzen bleibt verborgen. Die mangelnde Orientierungsmöglichkeit erzeugt ein Gefühl des Ausgeliefertseins und des Defaitismus; produktives Menschentum vollzieht sich dagegen im Vorgang sinnbezogener rationaler Orientierung innerhalb der Welt. Da der Anpassungszwang der formalrationalen, aber die Vernunft nicht inhaltlich, als schöpferisches Movens des Menschen, freisetzenden Gesellschaft minutiös ist und Arbeitsprozeß wie Freizeit in gleicher Weise umfaßt, gewinnt sie den Charakter naturwissenschaftlicher Determiniertheit. Seit Buckles History of Civilization in England ist die Erscheinung deutlich erkannt. In dieser Gesellschaft nun lebt der Mensch, ohne seiner selbst gewärtig zu werden, ausgeliefert; das Problem ist überall dort noch komplizierter, wo die mangelnde Orientierungsmöglichkeit des Menschen nicht durch eine umfassende ideologische Sinngebung überdeckt werden kann, als einer Art Ganzheitsprinzip. Die soziologischen Grundlagen, auf denen die Demokratie seit der glorious revolution beruhte - vielfach verbunden mit der Geschichte der klassischen Bildung in Europa -, sind abgestorben. Es ist eine ernste Frage, ob die Demokratie im realen Verstande nicht lediglich als vorübergehende und nur eine historisch festlegbare Gesellschaftsklasse berührende Erscheinung angesehen werden muß. Das relativ einheitliche Selbstverständnis der bürgerlichen Klasse als Träger der demokratischen Entwicklung, beruhend auf der Erfahrung des bewußten Existenzrisikos und des Verantwortungszwanges, somit der Erfahrung realer Freiheit, erschien losgelöst von dem Hintergrund weithin unbewußten Lebens. Der Verlust der ökonomischen Selbständigkeit zwang diese Klasse in den Prozeß ihrer inneren Auflösung bei gleichzeitigem Eintritt der Massen in ihr Bewußtsein. Bewußtes Leben aber bleibt nun jenem Grad von Bewußtsein verhaftet, dessen die Industriege- 
sellschaft bedarf, um ihr Funktionieren sicherzustellen, Bedürfnisse zu wecken und ideologische Surrogate zu vermitteln; dem Verfall des humanen Selbstbewußtseins der bürgerlichen Schicht entspricht kein Gewinn dieses Selbstbewußtseins im ganzen. Das entwickelte Gesamtbewußtsein bleibt partiell, bei ökonomischer Abhängigkeit; partielle Rationalität im adäquaten Verhältnis zur Teilwirklichkeit. Selbst in Ländern starker bürgerlich-liberaler Tradition sind die daraus resultierenden Probleme unverkennbar; die in den Massen aufgestauten Aggressionstriebe als Ausdruck unerlöster Menschlichkeit werden zwar durch die Zwänge einer funktionierenden und mit einem hochentwickelten Lenkungsinstrumentarium versehenen Wirtschaft neutralisiert, können jedoch jederzeit, und fast widerstandslos, verselbständigt werden. Die Perspektivenlosigkeit des Lebens, leere Rotation, seine gleichzeitige Unfaßbarkeit, die Verkrüppelung des Menschen, sammeln Leid an, dunkel und unbegriffen, bereiten den Ausbruchsversuch. Auf den Widerspruch von Pseudorationalität und Akkumulation irrationalistischer Bestände hat Herbert Marcuse zu Recht aufmerksam gemacht. Sie sind jederzeit aktivierbar, unter mythischen Symbolen, den Totempfählen des Anfangs. Die Kruste ist sehr dünn, die den Untergrund verbirgt, unter der eine Eruption Ausweg sucht. Es gibt eine unsägliche Bedürftigkeit des Menschen in diesen Extremen des Widerspruchs, Bedürftigkeit nach dem Menschsein, eine noch stumme Revolte. Der potentielle Ausbruchsversuch aber ist ohne Bindung und jeder Perversion preisgegeben.

Der Widerspruch zwischen der Rationalität von Anpassungszwängen und unerfüllter Menschlichkeit kann tödlich werden; im System der Bedürfniserzeugung wird der Mensch, seines Subjektseins entblößt, gerade noch gehalten. Bildung soll nun - und die Frage nach einer Veränderung der Gesellschaft an der Basis kann hier nicht gestellt werden -, Wirklichkeit einbeziehen, damit der Mensch ihrer mächtig werde, sie soll ihn denkend und handelnd mit ihr verbinden und so sein künftiges Verhältnis antizipieren, um es sinnvoll zu machen. Diese Voraussetzung, als Voraussetzung jedes realen Humanismus, ist unbestritten; unbestritten bleibt aber auch, was Ernst Bloch in "Subjekt-Objekt" unter Hinweis auf den Positivismus sagt: Die reine Tatsache sei das Dümmste, was es gibt. Schon die eben genannte Voraussetzung ist zubereitete Voraussetzung; alle Wirklichkeit ist zubereitet. Grad und Absicht der Zubereitung aber sind unterschiedlich erkennbar, je mehr Unmittelbarkeit vorgetäuscht wird, um so schwieriger wird es, kritisches Bewußtsein zu entwickeln. Wirklichkeit kritisch zubereiten zu helfen, Hilfe bei der Inbezugsetzung der Vielfältigkeit der Erscheinungen - an sich nur ein wüstes Chaos -, auf das humane Selbstverständnis ist Aufgabe der Bildung. Sie wird damit entscheidend zur Aufgabe der Abstraktion, der Schaffung von Distanz, der Herausnahme des Menschen aus der Verfügbarkeit, die Entlassung schöpferischen Widerspruchs. Dies gilt immer und überall, es gilt heute, angesichts des technischen Instrumentariums, durch das Verfügung über den Menschen bewirkt wird, im besonderen Maße. Es gilt von Beginn an, weil dieses Instrumentarium den Menschen vom Beginn an erfaßt; jede Fiktion eines isolierten Kinderlebens, jede These einer den Bildungsprozeß verzögernden Psychologie trägt zur intellektuellen Wehrlosmachung des Menschen und damit zu seiner Demoralisierung bei; sie trägt dazu bei, Wirklichkeit als menschlich-geistiges Phänomen auszulöschen. Distanz aber und Kategorien der Zuordnung, der humanen Zuordnung, vermittelt die klassische Bildung in hervorragender Weise. Es ist nicht behauptet worden, daß sie die einzig 
mögliche, nur daß sie die beste sei, und zwar gerade unter den Bedingungen der gegenwärtigen Existenz des Menschen. Es darf dies auch durch einen kurzen Vergleich mit dem Bildungswert der Naturwissenschaften erhellt werden; ohne den durch sie repräsentierten Bereich ist keine Allgemeinbildung heute denkbar. Auch die Naturwissenschaften beruhen auf dem Prinzip der Distanz; auch in ihnen wird sich das Bewußtsein selber zum Gegenstand. Ihre Beziehung zur Gegenwartsstruktur der Gesellschaft ist eminent und doch zugleich höchst vermittelt; vordergründige Bezüglichkeiten haben keinen Platz. Über die Naturwissenschaften führt demnach durchaus ein Weg zur Existenzerhellung, zur Erhellung des Strukturgefüges nach Ordnung von Gesetzen; das Zufällige bleibt ausgeschlossen. Der Wert dieser abgesonderten und strengen Erkenntnis ist unbestritten. Es war dennoch darauf hingewiesen worden, daß naturwissenschaftlich-technische Perfektion und prähistorische Ideologie bruchlos nebeneinander existieren können; die Grenzen der naturwissenschaftlichen Bildung werden damit offenbar, ihre Emanzipation aus einer ursprünglich humanen Bindung. Die naturwissenschaftliche Bildung muß auch im humanistischen Gymnasium ihre feste Stätte haben; Aufgabe der klassischen Bildung ist es jedoch, den anfänglich einheitlichen Ursprung des naturwissenschaftlichen und des humanen Denkens in diesem Zusammenhang aufzuweisen - exemplarisch am Beispiel des antiken Selbstverständnisses -, und das daraus resultierende Problembewußtsein in die Realität des modernen Menschen zu übertragen.

Ein letzter Umweg: Das Verhältnis von Schule und Lebenswirklichkeit war eines der entscheidenden Probleme, von dem die pädagogische Reformbewegung um die Wende des Jahrhunderts ihren Ausgang nahm; auf dem Hintergrunde einer Ideologie, die Elemente von Rousseau bis Nietzsche umfaßt, hatte sie sich dem Bewußtseinsidealismus entgegengestellt, um das Unmittelbare freizusetzen. Die Fragwürdigkeit dieses Begriffs ist bereits dargetan worden und der in ihm enthaltene Widerspruch. Ein anderes kam hinzu: Die moderne Gesellschaft ist abstrakt, ihre Mechanismen werden nur einer methodisch sorgfältigen Analyse deutlich. Die für die Zukunft bedeutsamsten Formen der industriellen Arbeit sind über den Bildungsprozeß nicht mehr reproduzierbar; die Wirklichkeit der gesellschaftlichen Arbeit muß zudem in der Schule immer eine Fiktion bleiben, da sie unter ihren realen Bedingungen, im Koordinatensystem der ökonomischen Zwänge nicht erlebt und der in ihr wirksame Prozeß der Entfremdung nicht Gegenstand des Bewußtseins werden kann. Noch Marx glaubte, dabei seine Herkunft aus Aufklärung und Idealismus im Sinne der qualitativen Sonderheit des Menschen nie verleugnend, daß über die Teilnahme am gesellschaftlichen Arbeitsprozeß die Grundelemente aller entscheidenden Produktionszweige erkannt sowie die Entfremdung des Menschen erfahren werden könne; Bedingung bleibt bei ihm, dies sei hinzugefügt, eine strenge und weitergeführte Allgemeinbildung im Sinne der klassischen Bewußtseinsbildung. Gerade hier wird deutlich - da es sich bei der industriellen Wirklichkeit um die entscheidende Lebenswirklichkeit handelt -, wie sehr sich die Voraussetzungen geändert haben. Die Erkenntnis der Grundbedingungen der Produktionszweige, damals noch stark auf dem Handwerk beruhend, ist unvergleichlich komplizierter geworden, die Entfremdung des Menschen, dort noch unlösbar von seiner sichtbar werdenden materiellen Not, bleibt ohne Erkenntnis der im Unterbewußtsein hockenden Ängste unmöglich. Marxens bedeutsamer Versuch, Schule und Lebenswirklichkeit miteinander 
zu verbinden, zielte jedoch darauf ab - und dieser Exkurs sei gestattet -, den Bildungsprozeß in Schule und gesellschaftlicher Produktion gleichzeitig durchzuführen und beide, unter dem Aspekt der Bewußtseinsbildung, aufeinander zu beziehen; er behält, unter Beschränkung seines Anspruchs, eine bleibende Bedeutung, doch kann das hier nicht dargetan werden. Die Fragestellung geht somit dahin - und dies ist mit polytechnischer Bildung, von Marx technological training genannt, angezeigt -, an welcher Stelle der geschlossene Kreis der Schule zum Leben hin geöffnet werden, wie aus der Schule gleichzeitig in das Leben hinausgetreten werden kann; niemals geht es um das Hineinnehmen einer vorgegebenen Lebensunmittelbarkeit in die Schule selbst. Die Forderung nach Einbeziehung des Lebens in die Schule als Erzeugung von Arbeitsweisen oder Einübung politisch-gesellschaftlicher Formen innerhalb ihres geschlossenen Kreises ist vielmehr über die Anstöße der modernen Reformbewegung erfolgt und gilt als Antithese einer primär auf der strengen und systematischen Entfaltung der Erkenntnisfähigkeit beruhenden Bildung, wie sie der klassischen eigen ist. Hier soll nun eine Abgrenzung vorgenommen werden, auch auf die Gefahr eines Mißverständnisses hin. Im Bildungsproze $ß$ muß dem Menschen Gelegenheit zur Bewährung im Tun, dem Umfang seiner Erkenntnis gemäß, gegeben werden; dies ist unbestritten. Sie hat innerhalb der Möglichkeiten zu geschehen, die die Schule real anbieten kann. Auf die feste Beziehung von Handeln, Erkenntnisumfang und realer Verantwortung muß streng geachtet werden; die Gründe des Handelns müssen in das Bewußtsein des einzelnen genommen werden, sie dürfen vom Erkenntniszwang nicht gelöst sein. Dies ist Voraussetzung für das Wachsein des Geistes im Tun, Würde der Freiheit. Die These, die Schule sei eine Art Gesellschaft im Kleinen, das Leben der Erwachsenengesellschaft könne in ihr beispielhaft geübt werden, hält keiner Nachprüfung stand. Struktur und Motivation der Gesellschaft können in der Schule nicht reproduziert werden, weder die Triebkräfte der Geschichte noch der abstrakte Mechanismus der Arbeitswelt. Dies alles ist viel zu ernst und steht außerhalb jeder Spielerei; über ihre Reproduktion im Spiel kann die wirkliche Welt nur verschlossen werden, sonst nichts. Für die Bereitschaft zum sittlichen Handeln in einer Wirklichkeit, die den heranreifenden Menschen erwartet, bleibt zudem das Vorbild des Lehrers entscheidend; ob er in dieser Wirklichkeit, die für den Schüler immer erst erwartete Wirklichkeit ist, handelt oder nicht, sich den Gewalten stellt oder sich vor ihnen verbirgt, geht als Erfahrung durch alle Jahre mit, auch wenn die Gründe zunächst nicht verstanden sind. Eben dies aber liegt außerhalb jeder systematischen Betrachtung.

Die Einübung von Verhaltensweisen schließlich, wie sie aus einem industriellen Bedürfnis abgeleitet werden, als Einstellungen, Mobilität des Denkens, als Anpassung an den Rationalisierungszwang, als jeweils wechselnd partielle Erfassung vorgegebener Sachverhalte, überläßt den Menschen der Manipulation. Ökonomisch vielleicht effektiver geworden, voreingewöhnt für den Produktionsapparat der Gesellschaft, ist er austauschbar von System zu System; Verfügung über ihn wird herausgefordert. Einübung ist Gegensatz von Bewußtheit, von Einsicht des Ich gegenüber der Zerreißprobe des Wirklichen.

Die klassische Bildung aber beruht darauf, daß Realität über Erkenntnis vermittelt wird, alles Handein bleibt an sie gebunden. In einer Zeit, in der die Inkommensurabilität von Schule und Wirklichkeit auf Grund objektiver Bedingungen immer größer 
wird, gibt sie den weiterwirkenden und verbindenden Anstoß. Sie nimmt den Menschen aus der Verhaftung heraus und macht Wirklichkeit zu seinem Gegenstand; sie läßt ihn schließlich, inmitten der Zwänge, Freiheit erfahren, die Tür wird aufgestoßen. Das ist nicht möglich ohne Kategorien der Zuordnung, kritisch-analytisch, protagoreisch, Vertreibung des ideologischen Nebels, unerbittliches Wachsein, aber auch konstruktivhoffend, Pneuma, veni creator spiritus. Es geht um die Handlungsfähigkeit für überdauernde Zwecke, den langen Atem, um das Unverfügbare am Menschen. In diesem Sinne läßt Bildung eigene Substanz erfahren, in der sich die Wirklichkeit herausgefordert sieht, öffnet sie über Erkenntnis den Schacht des Ich, legt sie Gegenwart bloß. Sie löst den Widerspruch der Existenz nicht auf, sondern macht ihn bewuBt, auch um den Preis, den Sokrates zahlte; sie läßt den Menschen nicht leben, indem sie ihm seine Würde nimmt. Sie geht, so verstanden, bis an die Grenze: In ihr gewinnt der Mensch zwar Substantialität gegenüber der Gesellschaft inmitten ihrer Versteppung, aber er wird zunächst in einem geschlossenen Raum auf sich seiber zurückgeworfen, allein in der kalten Luft der Freiheit, doch nicht ohne Möglichkeit der Verwandlung. „Den Schmerz wachzuhalten, das Verlangen zu erwecken, ist die erste Aufgabe eines jeden, den die Verdunkelung der Ewigkeit leiden macht ${ }^{\text {ty }}$, schreibt Martin Buber in den „Reden über Erziehung"; produktive Leidensfähigkeit als Ergebnis des in das Bewußtsein versetzten Widerspruchs ist Bestandteil jeder klassischen Bildung heute.

Die große Distanz schafft die Beziehung zum Werden als nie erledigte Aufgabe, sie hält Wirklichkeit für den Menschen offen. Geistige Erweckung über Distanz fällt der klassischen Bildung zu. Das humanistische Gymnasium täte gut daran, sich uneingeschränkt zu seinem Bildungsauftrag zu bekennen; es repräsentiert ein Stiick bester deutscher Geschichte. Es hat eine unersetzliche Aufgabe, am Beispiel jener ersten und wirkenden Erscheinung des Geistes, der Thomas soviel verdankt wie Marx, jenes unerhörte Spannungsverhältnis von Geist und Wirklichkeit, das menschliche Verhältnis, erfahrbar bleiben zu lassen, als schöpferische Hoffnung, Schutz vor Auslieferung. Der Mensch, in seiner Selbsterfahrung als Totum, der Erfahrung seines Bestimmtseins zur Sichtbarkeit, ist in der Antike erkennbar geworden; er bleibt Kontrapunkt aller Geschichte. Nicht Trost der Welt und imaginäres Reich, sondern zu Verwirklichendes. Dabei gibt es am Ende noch zu beachten:

Das humanistische Bildungsprinzip ist ohne Bindung an eine Klasse. Die bürgerliche Klasse als Bildungsschicht einer Epoche kommt nie zurück. Ihr Verwesungsprozeß, das Herauskriechen des Chaos aus ihrem Leibe, bildet den Gegenstand der deutschsprachigen Weltliteratur dieses Jahrhunderts. Es gilt, jede Kraft einzusetzen, um aus der Substanz, dem entfremdeten Leben, Zukunft zu entbinden; die Basis der klassischen Gymnasien ist brüchig geworden, sie trägt nicht weiter. Humanistische Bildung ist kein sublimes Glück der wenigen, sondern Frage nach dem Letzten als Kriterium der Humanität. Aus dem Begriff des Menschen, aus seiner Verwirklichung, ist niemand entlassen. In diesen Zeiten hat sich die Wahrheit des Auftrags zu erweisen; seines ursprünglichen gesellschaftlichen Ortes enthoben, ist das klassische Gymnasium freigesetzt wie nie zuvor. Humanistische Bildung ist realer Humanismus, indem sie das der menschlichen Möglichkeit inadäquat Gewordene fortwirft zugunsten des Menschen.

8 Buber, Martin: Reden über Erziehung, Heidelberg 1953, S. 76. 